\title{
Remotivation de l'article médiéval EN Spécificité de la Région Occitanie
}

\author{
Vincent Rivière \\ Chargé de mission catalan - occitan, Région Occitanie, Hôtel de Région, 22 boulevard du Maréchal \\ Juin, 31406 Toulouse Cedex 9 \\ vincent.riviere@1aregion.fr \\ Chargé de mission linguistique, Congrès Permanent de la Langue Occitane, Château d'Este Av. de la \\ Pléiade F-64140, Billère cedex \\ v.riviere@locongres.org \\ Membre associé, CLLE-ERSS UMR 5263, Université Toulouse Jean Jaurès, 5, allées Antonio- \\ Machado 31058 Toulouse Cedex 9
}

\begin{abstract}
Résumé
La microtoponymie de la zone L'Isle-Jourdain (30 km de Toulouse en zone gasconne) est largement caractérisée par la présence de $E N+X$ qui remonte au nom du propriétaire de la ferme précédé de la particule honorifique $E N$. En tant qu'article personnel, nous nous attendons à ce que $E N$ soit utilisé uniquement devant des noms de personne (En Martin, En Colau, etc.). L'usage de $E N$ devant un autre syntagme qu'un nom de personne tel qu'un nom de métier comme En Gireta suggère deux hypothèses :
\end{abstract}

- $\quad$ Soit, dans En Gireta, Gireta est un nom de personne dérivé d'un nom de métier et $E N$ peut encore être article au moment de la formation du toponyme.

Soit, dans En Gireta, Gireta est un nom de métier et suppose que EN est réanalysé comme préposition signifiant «chez», synonyme de entà en gascon toulousain.

L'analyse de la variation diachronique en quatre étapes peut permettre d'expliquer la deuxième hypothèse où l'article personnel médiéval est remotivé en préposition locative après desémantisation.

\footnotetext{
Abstract

Remotivation of the medieval article EN - Specificity of the Occitanie Region. The microtoponymy of the zone of L'Isle-Jourdain $(30 \mathrm{mn}$ of Toulouse in gascon zone) is strongly marked by de the presence of $E N+X$ which goes bauck up in the name of the owner of the farm preceded by the honorary particle $E N$. Es personal article, we expect that is only used in front of names of people (En Martin, En Colau, etc.). The use of $E N$ in front of a sintagm other than a personal name such as a job like En Gireta suggest two hypotheses:

- $\quad$ Or in En Gireta, Gireta is a name of someone derived of a name of job and can be still article at the time of the formation of the toponym.

- $\quad$ Or in En Gireta, Gireta is a name of job and it supposes that $E N$ is reanalyzed as preposition in the meaning of "entà" in the sense of Toulouse (else's house).

The analysis of the diachronic variation in four stages can help explain the second hypothesis where the medieval personal item is re-motivated in preposition of place after desemanticising process.
} 
Les recherches que j'ai menées, en admettant qu'elles s'appuient sur un échantillon représentatif de la zone de présence de l'article médiéval EN, telle que la décrit Nègre (1955), m'ont permis d'établir une liste de quelques 65 microtoponymes, sur 330 collectés. Cette recherche s'est limitée à la commune de L'Isle-Jourdain qui a une superficie de 7048 hectares. Celle-ci fait suite à une enquête de terrain et comporte de nombreuses attestations orales filmées, datées et consultables. De plus, chaque microtoponyme a été décrit sur une fiche spécifique.

Ces fiches sont composées d'un tableau comprenant les attestations écrites, datées des plus anciennes au plus récentes. Ces dernières sont issues des cadastres, de la Carte Cassini, de l'IGN, du service du feu, de l'armée, etc. Le Dictionnaire topographique de Polge (seconde moitié du $\mathrm{XX}^{\text {ème }}$ siècle, sous forme de fiches papier consultables au Archives départementales du Gers) est répertorié d'une part, dans le tableau de façon synthétique, d'autre part, de façon complète, à la suite du tableau.

Sont ensuite répertoriées les attestations orales datées, transcrites, puis les définitions en langue moderne (Mistral, 1979 ; Palay, 1991) et les étymologies du dictionnaire FEW (Wartburg, 1928 -2003).

De nombreuses autres sources toponymiques ou anthroponymiques complètent ces données.

La première occurrence à laquelle nous allons nous intéresser est le nom de la commune qui a suscité bien des interrogations et bien des recherches (Rivière, 2018), du fait que deux formes sont toujours présentes en français à l'oral, L'Isle En Jourdain et L'Isle-Jourdain, et une seule en occitan : L'Isla de Baish. Contre toute attente, alors que cet article est une caractéristique majeure de la microtoponymie occitane de cette zone (voire française, $80 \%$ de la microtoponymie du Sud de la France étant occitane), ce dernier est absent du nom occitan de la commune et régulièrement présent dans le nom français de cette dernière.

\section{Le nom de la commune : L'Isle-Jourdain / L'Isle En Jourdain}

Le nom officiel (français) de la commune que j'ai choisi comme échantillon est aujourd'hui L'Isle-Jourdain.

Selon Bourse \& Laborie, cette commune a porté bien des noms :

[Mutatio bucconis], (Isc) [Ictium], La Ysla, La Hilha, Insula, La Ylha en Jorda, Insula Jordani, Lille Jourdain, Lisle Jourdain, L'Isle en Jourdain, L'Isla-de-baisch, L'Isle-Savès, L'Isle sur Save. Bourse \& Laborie $(1995,62)$

Polge (1960, 93), donne des précisions sur les dates de présence ces formes dans un article consacré à l'analyse des noms de communes composés de L'Isle. Un de ceux-ci nous intéresse particulièrement. En effet, le nom de commune lui-même a contenu cet article pendant plus de quatre siècles, comme le confirme Nègre.

Il était normal qu'en ajoutant à un nom de lieu un nom de personne comme déterminatif, on le fît précéder du En honorifique. Ainsi [...], nous trouvons en 1580, l'Isle-en-Jourdain (GE), que l'on traduisait en latin Villam Insule Jordanis. ${ }^{\mathrm{i}}$ [...]. Nègre $(1955,24)$

Polge va également dans le sens de l'hypothèse d'une particule honorifique au sein de ce toponyme :

La composition peut n'être soulignée que par un trait d'union (Isle-Jourdain); [... ; enfin ce peut être aussi la particule honorifique en (Isle-en-Jourdain à côté de 1'Isle-Jourdain) qui 
représente dominu et non in comme se l'imagine un collaborateur de la Revue internationale d'onomastique. Polge (1960, 91)

Ce n'est qu'à la fin du $\mathrm{XX}^{\mathrm{ème}}$ siècle que la forme comprenant l'article a disparu de la signalétique (Rivière, 2018).

Polge et Nègre posent l'hypothèse selon laquelle la particule EN est un article honorifique, issu de l'évolution du latin DOMINU.

L'étude historique de Saverne renforce celle de Bourse \& Laborie concernant le syntagme spécifique Jourdain :

Suivant le plus grand nombre, au commencement du XIIe siècle, Raymond, fils aîné d'Atton d'Illa ou d'Isle, prenant part à la première croisade avec le Comte de Toulouse Raymond IV, son suzerain et son parent, eut, durant son séjour un fils qui fut baptisé dans les eaux du Jourdain. Ce fils ajouta à son nom celui du fleuve où il avait reçu les eaux du baptême et le laissa, ainsi augmenté, à ses descendants et à la ville qu'il bâtit autour du château d'Ictium. Saverne $(1914,1)$

Ces précisions sur le nom de ce seigneur laissent peu de place au doute concernant la nature $\mathrm{du} 3^{\mathrm{e}}{ }^{\mathrm{eme}}$ syntagme et renforcent, de fait, l'hypothèse de l'article honorifique. Néanmoins, le complément qu'apporte ce même historien sur la présence de l'article en question mène à penser qu'il en a une compréhension différente.

On lit tantôt dans les vieilles chartes : l'Isle-en-Jourdain, et tantôt : l'Isle-Jourdain. Exprimée ou sous entendue, la préposition «en» implique une idée de seigneurie, de possession, de main mise. C'est comme s'il y avait : l'Isle à Jourdain ou à Jourdan ou à Messire Jourdain. Saverne $(1914,2)$

Saverne semble comprendre cette syntaxe différemment de Polge et Nègre. Il semble en effet ajouter la notion d'appartenance.

Comme Pujaudran s'est construit avec deux syntagme «Pueg Audran » (générique Pueg (colline) + spécifique Audran (anthroponyme)), ou Montauban - Mont Auban - L'Isle En Jourdain a suivi cette construction, mais avec l'insertion de l'article personnel.

Le nom sans préposition, avec ou sans article, est équivalent à une forme moderne avec « de », comme Mont de Marsan. C'est le cas régime à valeur de génitif par juxtaposition, comme l'indique Jensen (1994, 12). Etymologiquement, $E N$ ne semblait pas être une préposition comme «à » qui exprime actuellement une appartenance. Que le nom Jourdain / Jordan ait été ramené de Jérusalem, ou bien qu'il ait déjà été un nom de famille ou d'homme, nous indique que le village qui s'appelait L'Isle / L'Isla a pris le nom du seigneur. Dans ce cas, la thèse de Nègre qui parle de $E N$ honorifique se vérifie parfaitement. Las Leys d'Amors parlent, à propos de $N A$ et $E N$, « d'habitude propre » ou « d'habitude honorable », par opposition à «l'habitude commune » qui est l'article du nom commun :

Tres son habitutz proprias, en autra maniera dichas honorablas, sos assaber: En, Na, An, coma : En Jacmes, Na Huga, An Bertran. E son dichas honorablas, quar segon nostra maniera de parlar, alcuna honor dona hom a persona can ditz En Garnier [...], e non ges can ditz : Garnier. Anglade $(1919,60)$

Salvat, dans sa Gramatica Occitana, suit l'hypothèse d'Anglade en affirmant que sa place est devant un nom d'homme, mais il utilise une terminologie différente : article personal (article personnel).

La formas En e Na, emplegadas autres còps dabant los noms pròpres d'òme e de femna, èran un vertadièr article personal. La fòrma En sola es emplegada auèi, e dins qualques dialèctes solament. Salvat $(1943,22)$ 


\section{Présence de l'article en Gascogne Toulousaine}

Les microtoponymes qui sont composés de l'article sont très nombreux dans cette commune. Sur 330 toponymes relevés, environ 65 le contiennent, soit presque un quart. Nègre décrit cette présence à partir de la «Carte d'état-major» :

Il existe deux aires de noms de lieux commençant par En: l'une à l'ouest de Toulouse, comprend le centre et l'est du département du Gers et déborde un peu sur le Tarn-et-Garonne et la Haute-Garonne. La seconde aire, à l'est de Toulouse, couvre le Lauragais (HauteGaronne et Aude) et la partie sud-ouest du département du Tarn. Entre les deux, la plaine de la Garonne est dépourvue de En [...]. Nègre $(1955,19)$

Ce chercheur a illustré ce propos par deux cartes qui décrivent, de façon schématique, la densité de cette présence sur les deux aires citées. Voici la carte schématisant l'ouest de Toulouse, où les croix représentent des groupes de 5 toponymes.

GROIPE DE LOLEST

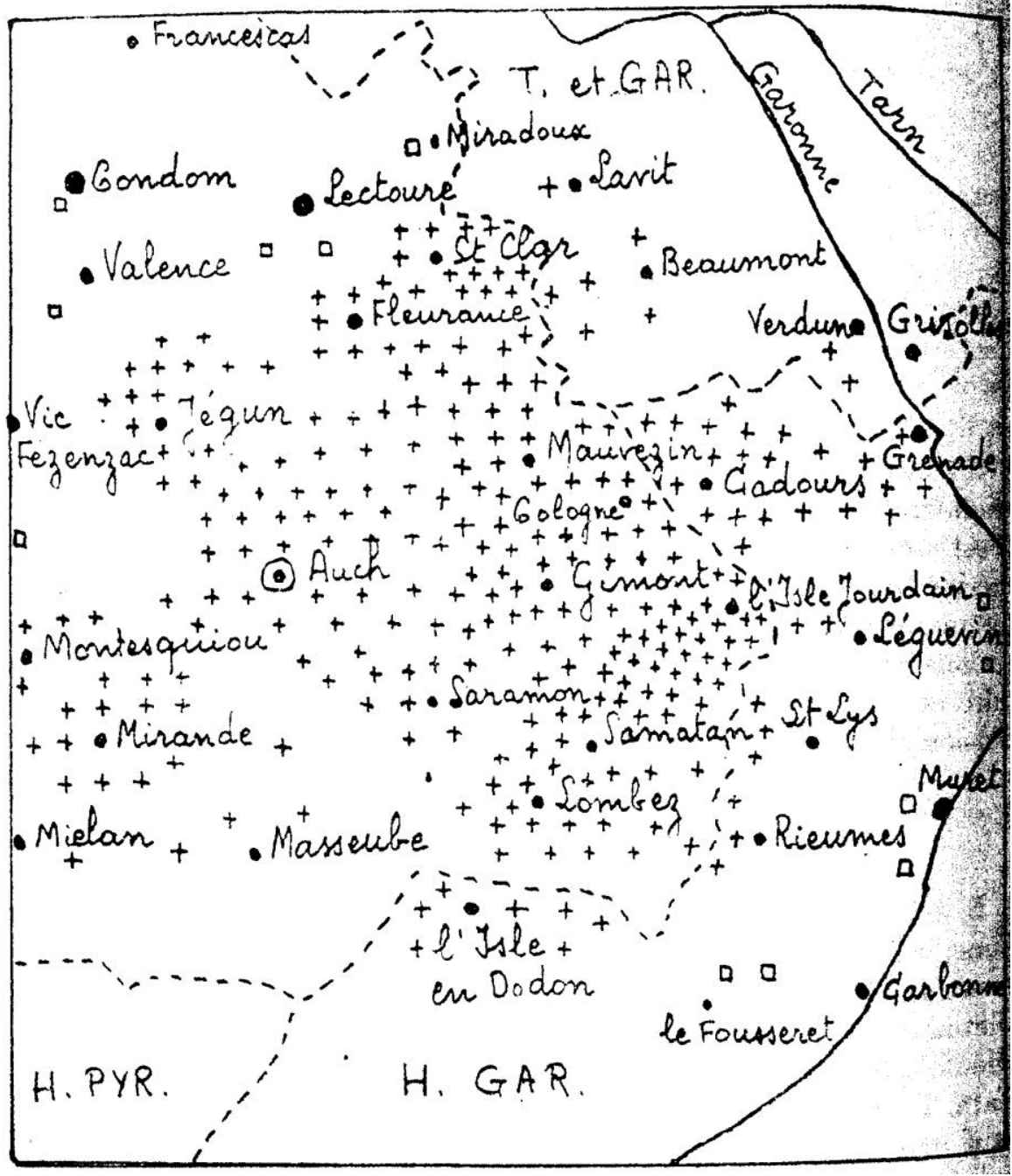

Nègre $(1955,19)$ 
Je vous présente la liste des formes que j'ai relevées en l'état, sans procéder à une correction graphique.

\begin{tabular}{|c|c|c|c|}
\hline Microtoponyme & Localisation & Microtoponyme & Localisation \\
\hline En balaguère & E4 & En girette & F4 \\
\hline En Barbarin (1640) & A-B3 & En gouardes & $\mathrm{C} 4$ \\
\hline En barbe & $\mathrm{C} 3$ & En Lauquet & D3 \\
\hline En barjaou & $\mathrm{B} 1$ & En licarre & D1 \\
\hline En bassières (En Baziège) & $\mathrm{C} 2$ & En magret & B4 \\
\hline En bazac & E7 & En mailloles $(1640-1805)$ & F3 \\
\hline En Bazac & F3 & En marot & B1 \\
\hline $\begin{array}{l}\text { En Bège / En Bèges } \\
(1640)\end{array}$ & $\mathrm{C} 2$ & En marquet & B5 \\
\hline En beoulaygue & $\mathrm{C} 2$ & En martin & B4 \\
\hline En berdot & B5 & En matalon & D2 \\
\hline En berduc & D3 & En Mesplet (1640) & B4 \\
\hline En bernadou & $\mathrm{C} 1$ & En millet & E5 \\
\hline En betpéou & E5 & En Montaut (1640) & D6 \\
\hline En bidalet (Embidaou) & G4 & En Nouailles (1805) & B3 \\
\hline En bladé & E4 & En nougaret (En nouret) & D7 \\
\hline En blans & $\mathrm{C} 2$ & En pejouan & D3 \\
\hline En Bornesian & D3 & En pelegrin & F3 \\
\hline En boubées & D7 & En péninon & D1 \\
\hline En bouscardéou & B4 & En Perdic & B2 \\
\hline En bragali & D3 & En perrin & D7 \\
\hline En cacharnaou & D2 & En Pertufet & F4 \\
\hline En Calotte & B3 & En peyret & B5 \\
\hline En candalle & E2 & En peyroutet & G4 \\
\hline En carbon & G4 & En pioque & H5 \\
\hline En carla & F3 & En Plante & B2 \\
\hline En Cazaux & C3 & En potico (poticaou) & F3 \\
\hline En Claous & A3 & En poulourine & D2 \\
\hline En claque & E4 & En rapeau & D5 \\
\hline En coulaou & E3 & En Robinau & D4 \\
\hline En coulin & B4 & En sissé & D1 \\
\hline
\end{tabular}




\begin{tabular}{||l|l||l|l||} 
En coulom & F4 & En superi & C3 \\
\hline En Coupes & C2 & En téoulet & E6 \\
\hline En Courtaou & B5 & En tiau & C6 \\
\hline En coustans & B5 & En Tocarlès & B2 \\
\hline En coustès & D6 & En toutoune & E3 \\
\hline En daran & B4 & En touzan & E4 \\
\hline En duguet $(1805)$ & D-E2 & En Trigoli & B5 \\
\hline En fourcet & E4 & En truyet & B2 \\
\hline En gaubert & D6 & En Verdure & A3 \\
\hline
\end{tabular}

La zone étudiée se situe à l'Est du département du Gers, à l'Est de la carte présentée cidessus également.

La carte d'état-major donne dans l'aire de l'ouest plus de 1.200 noms commençant par En, répartis entre 34 cantons. Les cantons de densité maxima sont : L'Isle-Jourdain, GE ${ }^{\text {ii }}$ (132 En), Gimont GE (111 En), [...]. La seule commune de l'Isle-Jourdain en compte $45 ;[\ldots]$. Nègre $(1955,19)$

Cette densité importante permet de travailler sur un échantillon varié qui est, en partie, représentatif de la diversité de cet article sur l'ensemble du territoire, et va nous permettre de tâcher d'en analyser la valeur en diachronie.

\section{Valeur de l'article}

\subsection{Article personnel honorifique médiéval occitan}

L'article défini a pour valeur la « définitude ». Il renvoie a un objet qui a déjà été évoqué : la prada (la prairie) (Jensen $(1994,61)$. Il est ainsi défini dans le sens où il a reçu une référence actuelle, pas seulement virtuelle : "nous devons parler de prairie». Certains objets ont une référence unique : le soleil. Dans ce cas, une seule référence est possible et elle est connue de tous. Les noms propres appartiennent à cette catégorie unica telle que la définit Jensen.

Les susbstantifs qui désignent des objets dont il n'existe qu'un exemplaire unique apparaissent le plus souvent au singulier : cel 'ciel' [...]. Les noms propres appartiennent par définition à la catégorie unica [...]. Jensen $(1994,25)$

Ainsi les toponymes n'ont généralement pas d'article (Auch, 32) ou bien ils ont des articles définis (L'Isle-Jourdain). Il reste une hésitation concernant les hydronymes, sans doute liée à l'influence du français sur l'occitan - Sava / La Sava - comme le confirme les enregistrements suivants :

Le veritable camin ende vénguer aqui, aqueth existava pas, passava le long de Sava e montava la petita còsta, e au cap de la petita còsta, i avèva La Costèra.

Fiche La Costèra : 00'52 [Campariol, 17/06/09, Toponimia l'Isla de Baish]

Totas Las Angladas es au bòrd de Sava.

Ficha La Costèra : 06’30, [Campariol, 17/06/09, Toponimia l'Isla de Baish] 
Òm se raprochava un petit pauc de La Sava, i avèva le Poton.

Ficha Le Poton : 02'15, [Caubet, 22/05/09, Toponimia l'Isla de Baish, Rivière])

Rivière $(2010,972)$

Le fait d'être en présence d'un article personnel de nom propre est donc peu habituel. Etant donné que les noms propres dépendent de la catégorie unica, l'article défini amène une information déjà présente comme dans «La Sava» par exemple. L'article honorifique ajoute une information supplémentaire, celle de valeur de seigneur ou plus simplement de reconnaissance. La recherche de Nègre nous servira de base pour déterminer les différentes valeurs potentielles qu'a pu connaître l'article EN :

D'abord, il faut remarquer que, dans les deux groupes, les noms en En désignent seulement des fermes, des hameaux, très rarement des paroisses ou des communes, comme Encausse (Cologne, GE). Ce fait suffit à démontrer qu'ils sont de formation récente. Il faut remarquer aussi que ces noms de lieux sont en très grand nombre des noms d'hommes précédés de $E n$. Ce sont des noms de baptême : [...]; ou leurs diminutifs ou augmentatifs : [...]; des noms de profession : [...], Empouticaou (L'Isle-Jourdain, GE); des sobriquets : [...]; des adjectifs d'origine : [...]; même des noms d'origine qui ont été des noms de personnes avant de redevenir noms de lieux : [...] Endaspet (Montpezat, Lombez, GE). Nègre (1955:22)

Il pose l'hypothèse selon laquelle ces toponymes sont, pour la plupart, des noms d'hommes précédés de l'article. Le locuteur de langue maternelle occitane que nous avons interrogé, nous a proposé une autre valeur pour cet article. Bien entendu, cette valeur se fonde sur le sentiment introspectif de cette personne, c'est un avis spontané sur cette forme. Aucune question ne lui a été posée sur la valeur de l'article. Il a simplement été demandé aux locuteurs, celui-ci n'est qu'une illustration, de lire la liste des microtoponymes présentée cidessus, puis de les prononcer selon leur habitude, de les localiser, voire de nous parler de ceux-ci.

Enbarjau, pasque le « En » se pronóncia [en]. En / Barjau, alavetz, sèi pas, pertot, planes son escrivuts en un sol mòt, Enbarjaou es escrivut en un sol mòt. Pensi que non qu'es pas vertat, que s'escrivèva en dus mòts, èra En / Barjau. Es a díser que, coma dirén " entau Barjau », pensi qu'èra en dus mòts e planes son botats en un.

Fiche En Barjau : 37’ 08, [Campariol, 17/06/09, Toponimia l'Isla de Baish]

Rivière $(2010,587)$

Ces enregistrements ont le mérite de démontrer que les locuteurs ont conscience d'une valeur potentielle de l'article. Celle-ci va dans le sens d'une réinterprétation de l'article comme préposition. Pour lui, cette "préposition» signifie l'habitation, le lieu où la personne vit. Nègre donne des précisions sur les variations diachroniques de l'article qui sont en pleine contradiction avec le sentiment du locuteur :

Ce fait que $\mathrm{En}$ et $\mathrm{Na}$ dans ces noms de lieux précèdent un nom de personne suffit à démontrer qu'il s'agit de En et Na particules honorifiques de l'ancien occitan, l'équivalent de Monsieur, Madame. [...] Cette survivance de En honorifique rend évident le sens des $E n$ des noms de lieux: les fermes portent aujourd'hui le nom d'un ancien propriétaire précédé de son titre honorifique. Cependant $E n$ n'est plus productif depuis un siècle : je n'ai pu trouver aucune ferme ayant pris le nom de son propriétaire depuis l'établissement de la carte d'état-major. [...] En et $\mathrm{Na}$ comme particules honorifiques étaient usuels dans les diverses parties du domaine occitan dès le XII ${ }^{\mathrm{e}}$ siècle, comme nous le voyons dans Les plus anciennes chartes en langue provençale de M. Brunnel. En était commun aux clercs, aux nobles, et aux manants de quelque importance. [...] Donc En des noms de lieux est la particule honorifique. Cela s'admet sans difficulté quand il est suivi d'un nom qui est manifestement un nom de personne. Mais comment l'interpréter quand il est suivi d'un nom qui est essentiellement un nom de lieu, comme dans En Barthe [...] ? Nègre $(1955,23)$ 
Afin de répondre à cette question, je propose une analyse diachronique de la variation de l'article en quatre étapes.

\subsection{EN, préposition locative ?}

\subsubsection{Quatre stades d'évolution possibles}

En tant qu'article personnel, nous nous attendons à ce que $E N$ soit utilisé uniquement devant des noms de personne (En Martin, En Colau, etc.). L'usage de EN devant un nom de métier suggère deux hypothèses :

- Soit, dans En Gireta, Gireta est un nom de personne dérivé d'un nom de métier et EN peut encore être article au moment de la formation du toponyme.

- Soit, dans En Gireta, Gireta est un nom de métier et suppose que EN est réanalysé comme préposition signifiant « chez », synonyme de entà en gascon toulousain.

Dès lors que nous envisageons que le deuxième syntagme n'est pas un nom de personne, l'analyse diachronique en quatre temps permet de prendre en compte cette hypothèse.

Stade 0: Présence du terme générique comme dans Anglada d'En Rapèu (Prairie de Monsieur Rapèu), (cf. Negre (1954, 104) : La Bastida En At, puis La Bastida d'En At, of. 'Labastide Dénat'). Dans ce cas, la forme avec la préposition «d'» ne serait qu'une évolution de la forme originelle Anglada En Rapèu (Prairie Monsieur Rapèu).

Stade $1: E N$ article personnel : uniquement $E N+$ nom propre de personne (prénom, nom de famille). En Pèr Joan (Monsieur Pierre-Jean) équivaut à « (a la bòrda) En Pèr Joan » (à la ferme Monsieur Pierre-Jean ». Dans ce cas, En Pèr Joan est un cas régime à valeur génitive qui dépend d'un nom générique antécédent sous-entendu.

Stade $2: E N$ préposition qui demande un complément de nom de personne (comme entà (chez)). Nous pouvons dire " entau Pèir, entau Nègre, entau haure » (chez Pierre, chez Nègre, chez le forgeron), mais pas * « entau bòsc / entà l'escòla / entà l'ostau 》 (chez bois / chez l'école / chez la maison). Si, réellement, il y a des noms de métier non anthroponymes, cela pourrait être la preuve de la remotivation de $E N$ article personnel, en préposition qui sélectionne un nom de personne.

Stade $3: E N$ préposition locative. S'il y a réellement des formes qui précèdent une partie du relief, de la flore ou des éléments minéraux tels que En Bòsc, En Comba, où bòsc et comba ne sont pas des noms de personnes, l'article serait devenu une proposition locative.

Pour répondre à cette problématique, $\mathrm{j}$ 'ai décrit tous les microtoponymes concernés de cette commune en établissant des classes sémantiques. Il est évident qu'aujourd'hui, pour les locuteurs, c'est communément le stade 2 qui est envisagé.

La formation de $E N$, article de nom de personne en occitan médiéval était une grammaticalisation et corrélativement une desémantisation : le mot DOMINUS, ou une évolution de celui-ci, qui était un élément lexical avec un sémantisme complexe (maître, seigneur, etc.), est devenu un mot grammatical, un article. Il n'a conservé de son sens premier que le fait qu'il dénotait une certaine forme de respectabilité du nom qui suivait.

La fiche En Barba (Rivière, 2010) nous livre des informations précieuses. Ces dernières sont contenues dans le second syntagme. La forme française actuelle est En Barbe. Comptetenu des erreurs multiples que l'on trouve dans les cartes, sans forme phonétique pour s'assurer de la forme réelle, deux significations demeurent possibles : la barba [b'arbo], faite de poils que portent certains hommes, et le barbèr [b'arbe], l'homme dont le travail 
consiste à raser la barbe des hommes. La prononciation actuelle [emb'arbo] nous invite à penser que c'est le premier sens qui est envisagé aujourd'hui. Le cadastre de 1640, détaillé par Polge nous donne, ceci étant, l'information suivante : «Mr Barbé tient borde en Barbé confronte septentrion chemin de Lisle à Mauvezin ». Cassini indique une forme semblable : «Enbarbé ». Ce n'est qu'à partir de 1846 que la forme actuelle apparaît. Le mot « barbèr » est de façon certaine le nom du métier. Mais comme indiqué ci-dessus, en 1640, il semble bien que cet homme ait déjà, comme nom de famille, ce nom de métier, qu'il a laissé à la ferme.

Le passage de En Barbèr, compris comme la maison qui a pour nom Seigneur Barbèr, cas régime à valeur de génitif, à En Barbèr, compris comme chez Barbèr, est un changement de valeur sémantique : $E N$ est passé du statut d'article au statut de préposition. L'évolution de Barbèr à Barba est probablement liée à la forme écrite officielle récente «Barbe ». On peut penser à un recul phonétique d'accent sur le modèle «Sant Anhan » [aj'a] $\rightarrow$ ['ano] «Sant Ànhan » (ou «Sant Anha ») : $\left[\operatorname{bar}^{\prime} \beta^{\prime} \varepsilon\right] \rightarrow\left[\mathrm{b}^{\prime} \operatorname{ar} \beta \mathrm{e}\right]$ et finalement, «Barbe » serait devenu Barba par attraction du mot barba, indiquant la pilosité masculine.

Le changement de sens de barbier à barbe indique que l'article a clairement perdu son sémantisme médiéval.

Sur l'échelle de Hopper \& Traugott (1993), nous pouvons considérer que l'évolution occitane $E N$, du latin DOMINUS, est passée d'un " content word », soit un lexème, avec un contenu sémantique autonome, à un simple mot grammatical qui est un proclitique. De fait, il n'a pas changé de « cline », soit de niveau sur l'échelle de Hopper \& Traugott. C'est un processus évolutif horizontal : il change de valeur sémantique, mais il ne descend pas sur l'échelle de grammaticalisation.

\subsubsection{EN devant des toponymes qui ne sont pas des noms d'homme}

J'ai classé les toponymes en cinq classes principales qui peuvent être divisées pour mieux les caractériser.

De toute évidence, la plupart des ces derniers contient des noms d'hommes, qu'ils soient de naissance ou de famille. Ils peuvent être entiers et simples, entiers et composés, hypocoristiques par aphérèse, hypocoristiques par suffixation ou par substitution de morphème final : En Carbon, En Colom, En Constans, En Gaubèrt, En Licarra, En Martin, En Pelegrin, En Tiau, En Pèr Joan, En Gaisha Arnaut, En Colau, En Berdòt, En Berduc, En Bernadon, En Vidalet, En Marquet, En Peirin, En Peiret, En Peirotet, En Calòta, En Colin, En Matalon.

Les noms de famille issus de régions, de villes, de métiers, de noms de Saints ou de minéraux composent la deuxième classe : En Basièja, En Daran, En Barbèr, En Bladèr, En Guardas, En Supèri, En Robinau, En Basac.

La troisième classe est celle des sobriquets : En Balaguèra, En Barbarin, En Barjau, En Beulaiga, En Bèthpèu, En Blancs, En Bragalí, En Magret, En Peninon, En Rapèu, En Teulet, En Tontona, En Trulhet.

Ces trois première classes viennent confirmer la thèse de $E N$ article honorifique personnel.

Ceci étant, certaines formes sont difficiles à intégrer dans l'une de ces classes. Je propose donc, avec prudence, trois classes ou sous-classes supplémentaires, pour lesquelles l'analyse est bien moins évidente que pour les trois premières.

- $\quad$ EN suivi de nom de métier : En Gireta, En Poticau 
- EN suivi de nom de flore, de minéral, de relief ou de bâti : En Malhòlas, En Mesplet, En Milhet, En Nogaret, En Planta, En Bobèas, En Bornesian, En Candala, En Carlar, En Costèrs, En Duguet, En Monthaut, En Noalhas, En Casaus, En Claus, En Cortau

- $\quad$ EN suivi de nom de faune : En Lauquet, En Marròt, En Piòca, En Perdic

La classe des sobriquets est difficile à maîtriser, et est un frein à la confirmation de l'hypothèse du Stade 3, car on ne peut écarter complètement que les microtoponymes des trois dernières classes soient des sobriquets. Néanmoins, aucune source n'indique qu'ils l'aient été.

La classe des noms de métiers correspond au stade 2.

Dans le cas des formes qui apportent une information topographique notamment, la thèse de la desémantisation et corrélativement d'une grammaticalisation, puis d'une remotivation en proposition locative est cohérente. Cela correspond au stade 3.

Voici quelques exemples de toponymes qui illustrent plus précisément cette hypothèse.

Les bobèas (boulbènes) sont des terres argilo-sablonneuses et se trouvent à côté d'un cours d'eau : Sava. Carlar peut-être un château ou un bâti perché sur une roche. Costèrs peut être lié au dénivelé, il peut également être un nom de métier : valet de chapelle. Ce toponyme se trouve au bout d'une côte importante. Duguet signifie « bord d'un cours d'eau (FEW, 1928) et il se trouve au ras d'un ruisseau. En Bornesian a été rasé avec l'arrivée de la mécanisation agricole, c'était un lieu où l'eau était abondante entre mares et ruisseaux. Astor (2002) nous indique que la racine prélatine Born signifie « qui s'attache au nom de sources et de rivières ».

Enfin, la classe comprenant des noms de bêtes - Lauquet (l'oison), Marròt (petit bélier), Piòca (dinde), Perdic (perdrix) - est difficile à faire rentrer dans quelconque étape de l'évolution diachronique, si l'on ne se résout à les intégrer dans la classe des sobriquets, bien qu'aucune définition ou attestation ne le confirme.

Compte-tenu de la productivité des sobriquets en microtoponymie, je ne peux affirmer que l'évolution diachronique en quatre étapes est absolument certaine. Néanmoins, exemples à l'appui, sur un simple échantillon de ces formes, représentatif en partie, cette hypothèse permet de caractériser les formes qui ne sont pas, de façon immédiate, liées à un nom d'homme. Par ailleurs, la corrélation entre le sémantisme et la réalité de terrain des derniers exemples va dans le sens de cette hypothèse que seul le sémantisme médiéval ne peut expliquer. 


\section{Appendice}

En Bobèas

\begin{tabular}{|c|c|}
\hline Reférence & Forme présente \\
\hline Saume de l'Isle 1298 (Polge) & $\varnothing$ \\
\hline \multicolumn{2}{|l|}{ Cadastre 1640 (Polge) } \\
\hline \multicolumn{2}{|l|}{ Cadastre 1666 (Polge) } \\
\hline Carta de Cassini $\left(18^{\mathrm{e}}\right)$ & $\varnothing$ \\
\hline \multicolumn{2}{|l|}{ Cadastre 1749 (Polge) } \\
\hline \multicolumn{2}{|l|}{ Cadastre début XIXe (Polge) } \\
\hline \multicolumn{2}{|l|}{ Cadastre 1845} \\
\hline Cadastre 1846 & En Boubées / Las Boubées \\
\hline Carta deu canton 1880 & Enboubées / Las Boubées \\
\hline \multicolumn{2}{|l|}{ Cénac Moncaut (Polge) } \\
\hline Carta deu servici de l'armada 1900 & En Boubées / Las Boubées \\
\hline Carta deu servici deu huec (début XXe) & Enboubées / Las Boubées \\
\hline Carta IGN 1948 & / Les Boubées \\
\hline Cadastre 1969 & Emboubées / Les Boubées \\
\hline Carta IGN 1970 & Les Boubées / Les Boubées \\
\hline Carta de la comuna 2002 & En Boubées / Les Boubées \\
\hline Carta de la comuna 2008 & En Boubées / Les Boubées \\
\hline Carta IGN 2008 & Les Boubées / Les Boubées \\
\hline
\end{tabular}




\section{Références bibliographiques}

ALIBERT, L. (2000). Gramatica occitana, segon los parlars lengadocian. Barcelona : I.E.O.-I.E.C, 530 p. [Réédition corrigée; $1^{\text {ère }}$ édition 1935].

ANGLADE, J. (1919-1920). Las leys d'amors : manuscrit de l'Académie des Jeux floraux, Toulouse : Privat, 4 vol., 203-186-184-187 p.

ASTOR, J. (2002). Dictionnaire des noms de famille et noms de lieux du Midi de la France, Millau : Éditions du Beffroi.

BEC, P. (1995). La langue occitane. Paris : PUF, Coll. Que sais-je ?

BEC, P. (1973). Manuel pratique d'occitan moderne. Paris : coll. Connaissance des langues.

BOURSE, R. (1992). «Isc (Ictium), un toponyme disparu au XIIe siècle », Isle était $n^{\circ}$ 7, 82-91

BOURSE, R. et LABORIE, G. (1995). L'Isle-Jourdain d'hier et d'aujourd'hui, Groupe Lislois de Recherches Archéologiques et Historiques, 272 p.

DAUZAT, A. (1951). Dictionnaire des noms de famille et prénoms de France, Paris : Larousse, 626 p. $\left[3^{\text {na }}\right.$ éd. $]$.

DAUZAT, A. (1949). Traité d'anthroponymie française : Les noms de Famille de France, Paris : Payot, $454 \mathrm{p}$.

FORDANT, L. (1999). Tous les noms de famille de France et leur localisation en 1900, Paris: Archives et culture, $1420 \mathrm{p}$.

GROSCLAUDE, M. (2003). Dictionnaire étymologique des noms de famille gascons Lescar: Per Noste.

HOPPER, P. et TRAUGOTT, E. (1993). Grammaticalization, Cambridge: Cambridge University Press, 1 vol., XXI-256 p. [rééditions : 1994, 1997, 2000, 2002].

JENSEN, F. (1994). Syntaxe de l'ancien occitan, éd. M. Niemeyer (Tübingen), coll. Beihefte zur Zeitschrift für romanische Philologie.

NÈGRE, E. (1977). Les noms de lieux en France, Toulouse. [2 ${ }^{\mathrm{e}}$ éd ]

NÈGRE, E. (1955). "En" et "Na" honorifiques au début des noms de lieux du Sud-Ouest, Extrait du "Bulletin philologique et historique" (jusqu'à 1718) du Comité des travaux historiques et scientifiques, 1953 et 1954, pp. 19-26.

NÈGRE, E. (1990-1991). Toponymie générale de la France, Genève : Droz, 3 vol. 1857 p.

NÈGRE, E. (1996-1997). «Quelques toponymes toulousains : 1. rue Joutx Aigues ; 2. Le Férétra ; 3. Le Bazacle ; 4. Place Saintes Scarbes ; 5. Cuisines ; 6. Basse Cambe », L'Auta, 1996, 308310; 1997, 18-22; 46-51.

PALAY, S. (1991). Dictionnaire du béarnais et du gascon modernes, CNRS Éditions, Escole Gastou Febus, 1053 p. [ed. orig. 1932, $2^{\text {da }}$ ed. 1963].

POLGE, H. (1960). «De quelques noms de communes gersoises », Bulletin de la Société archéologique du Gers, 91-100.

POJADA, P. (2009). Répertoire toponymique des communes de la région Midi-Pyrénées. Portèth de Garona : Loubatières.

RIVIERE, V. (2018). L'Isle-Jourdain/L'Isla de Baish (Gers) - Paradoxe du double effacement d'un marqueur toponymique majeur. Société Française d'Onomastique, Toulouse. (à paraître) 
RIVIERE, V. (2010). Estudi d'un contact dialectau : Le gascon orientau, Thèse de Doctorat. Toulouse, $1039 \mathrm{p}$.

ROHLFS, G. (1977). Le gascon : étude de philologie pyrénéenne. Pau : Marrimpouey jeune. [3 $3^{\text {ème }}$ éd.]

RONJAT, J. (1930-41). Grammaire historique des parlers provençaux modernes. Montpellier: Société des langues romanes.

SALVAT, J. (1998). Gramatica Occitana, Toulouse : Collège d'Occitanie, [1 $1^{\text {ra }}$ éd 1943]

SAVERNE, J. (2001). L'Isle-en-Jourdain et son histoire, Nîmes : éd Lacour. [ed. originale : Gimont, 1914].

SAUZET, P. (2004). Variation des finales occitanes et format de la syllabe. Nouveaux départs en phonologie : les conceptions sub- et suprasegmentales, 33-48. Trudel Meisenburg \& Maria Selig eds, Tübingen, Gunter Narr.

SAUZET, P. (1993). Attenance, gouvernement et mouvement en phonologie. Les constituants dans la phonologie et la morphologie de l'occitan. Montpellier: CEO/UPV 505 p.

SAUZET, P. (1974). Approches de la phonologie et de la morphologie d'un parler occitan: le dialecte de Sumène. Gard : Mémoire de maitrise, Université de Poitiers.

SEGUY, J. (1973). Atlas linguistique et ethnographique de la Gascogne. Paris : CNRS.

SUMIEN, D. (2009). Classification des dialectes occitans. Linguistica Occitana 7. (www.revistadoc.org).

WARTBURG, W. (1928-2003). Französisches Etymologisches Wörterbuch : eine Darstellung des galloromanischen Sprachschatzes publié par Jean-Pierre Chambon, Jean-Paul Chauveau, Bonn : F. Klopp, 25 vol.

\footnotetext{
'Hist. de Languedoc, XII, col., 1330 ; VIII, col. 704.

ii Gers
} 